\title{
Bright-Field STEM Tomography of Blood Platelets in Thick Sections
}

\author{
R.D. Leapman ${ }^{1}$, M.A. Aronova ${ }^{1}$, J.D. Hoyne ${ }^{1}$, G.N. Calco ${ }^{1}$, B.C. Kuo ${ }^{1}$, Q. He ${ }^{1}$, I.D. Pokrovskaya ${ }^{2}$, \\ L.J. MacDonald ${ }^{2}$, A.A. Prince ${ }^{2}$, B. Storrie ${ }^{2}$ \\ ${ }^{1}$ National Institute of Biomedical Imaging and Bioengineering, NIH, Bethesda, MD 20892; \\ ${ }^{2}$ Department of Physiology and Biophysics, University of Arkansas for Medical Sciences, Little \\ Rock, AR 72205
}

By performing electron tomography in the scanning transmission electron microscope (STEM), it is possible to obtain 3D reconstructions at a resolution less than $\sim 10 \mathrm{~nm}$ from stained plastic-embedded sections of eukaryotic cells in 1-2 $\mu \mathrm{m}$ thick sections. This is achievable because there are no imaging lenses after the specimen when the electron microscope is operated in STEM mode, so that chromatic aberration of the objective lens does not compromise the spatial resolution when there is strong multiple inelastic scattering [1-4]. However, to image thick sections it is necessary to avoid a second source of resolution loss due to geometrical broadening of the probe, which can be mitigated by selecting a small probe convergence angle of $\sim 1 \mathrm{mrad}$. Previous work has also shown that by using an axial bright-field detector instead of a standard high-angle annular dark-field detector, it is possible to reduce a third source of resolution loss caused by multiple elastic scattering in the lower part of the specimen [2-4].

Here, we have applied axial bright-field STEM tomography to determine the 3D ultrastructure of human blood platelets, which are small anucleate blood cells that aggregate to seal leaks at sites of vascular injury and are important in the pathology of atherosclerosis and other diseases. Of particular interest are the morphological changes that occur in $\alpha$-granules, which contain important proteins released when platelets are activated [5]. Due to difficulty in controlling the physiological state of platelets, structural changes that occur in the early stages of $\alpha$-granule activation are not yet fully understood.

Electron tomograms were acquired using an FEI Tecnai TF30 transmission electron microscope equipped with a field-emission gun and operating at an acceleration voltage of $300 \mathrm{kV}$. The instrument was equipped with a Gatan bright-field STEM detector. Blood drawn under IRB procedures was incubated for 5 minutes and treated with prostacyclin and apyrase to suppress further activation before being high-pressure frozen at 2100 bar. Samples were freeze-substituted with osmium tetroxide and glutaraldehyde, dehydrated with acetone and embedded in epon. Sections were cut to a thickness of $1.5 \mu \mathrm{m}$ and stained with uranyl acetate and lead citrate, before being coated with carbon and 20-nm gold nanoparticles, which served as fiducial markers. Dual axis bright-field STEM tilt series were acquired over an angular tilt range of $\pm 68^{\circ}$ with a $2^{\circ}$ tilt increment. Tomograms were reconstructed using the IMOD program [6] and surface rendered using FEI Amira 3D software.

An orthoslice through a STEM tomogram from a $1500 \mathrm{~nm}$ thick section of a blood platelet in early stage of activation in Fig. 1A shows tubules extending from a decondensing $\alpha$-granule to the plasma membrane, whereas other $\alpha$-granules remain in their condensed state, as seen in the $3 \mathrm{D}$ visualization in Fig. 1B. The internal arrangement of organelles is shown in more detail in Fig. 2A and plasma membrane in Fig. 2B reveals numerous pores from the tubular extensions of the $\alpha$-granules [7, 8]. 
References

[1] A.E. Yakushevska et al, J. Struct. Biol. 159 (2007) p. 381.

[2] M.F. Hohmann-Marriott et al, Nature Methods 6 (2009) p. 729.

[3] A.A. Sousa et al, Ultramicroscopy 109 (2009) p. 213.

[4] A.A. Sousa et al, J. Struct. Biol. 174 (2011) p. 107.

[5] J. Kamykowski et al, Blood 118 (2011) p. 1370.

[6] J.R. Kremer, D.N. Mastronarde, J. Struct. Biol. 116 (1996) p. 71.

[7] L.J. MacDonald et al, (2015) in press.

[8] Research supported by the intramural program of the National Institute of Biomedical Imaging and Bioengineering, NIH. Work in the Storrie laboratory was supported in part by NIH grant R01 HL119393.
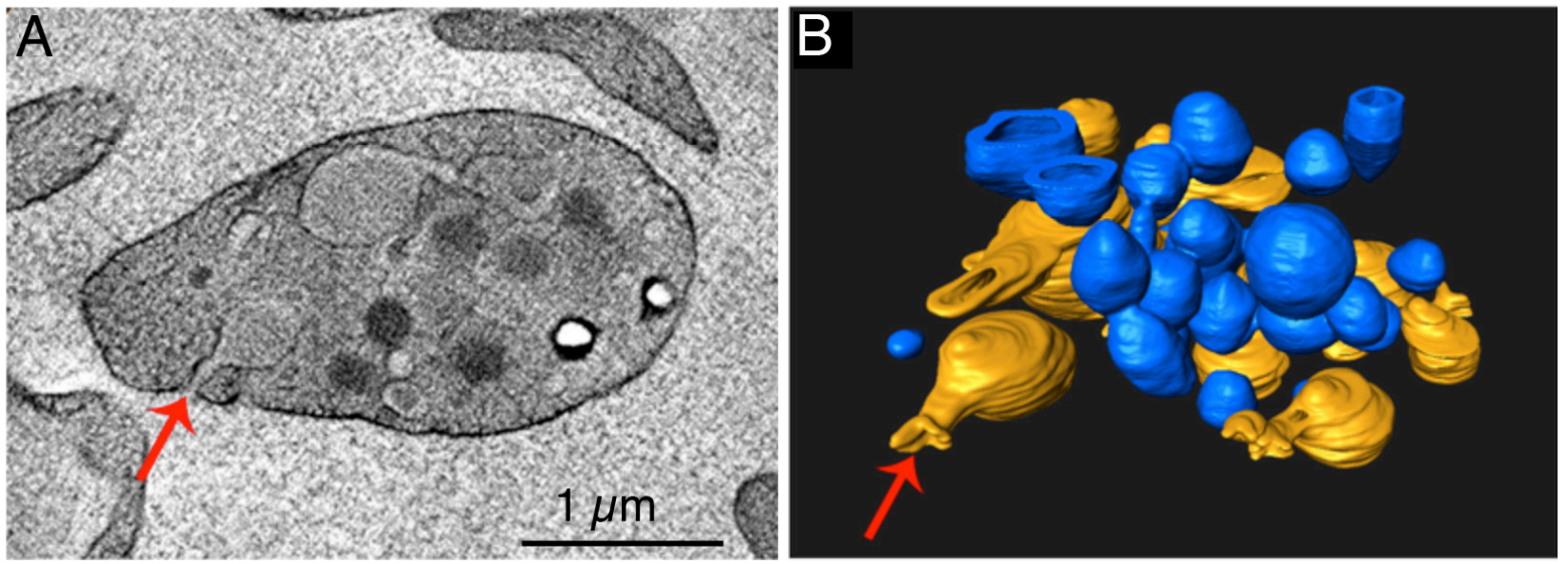

Fig. 1. STEM tomography of $1500 \mathrm{~nm}$ thick section of plastic-embedded, frozen and freezesubstituted human blood platelet in early stage of activation: (A) orthoslice showing tubular extensions from decondensing $\alpha$-granule (red arrow); (B) 3D model showing condensed unactivated granules (blue) and decondensing activated granules (brown). Adapted from reference [7].
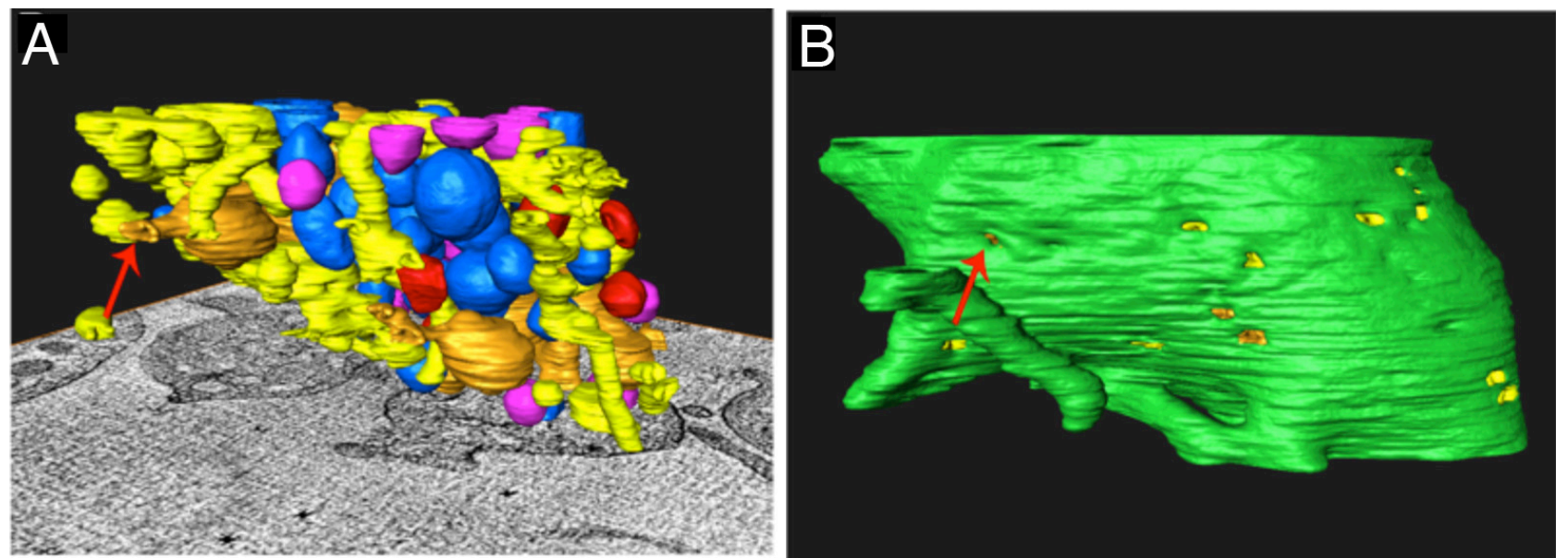

Fig. 2. Surface rendered models of platelet in early stage of activation: (A) internal structure of organelles showing canalicular system (yellow), condensed $\alpha$-granules (blue), decondensing $\alpha$ granules (brown), dense granules (red), and mitochondria (purple); (B) plasma membrane (green) showing openings of the internal tubular extensions originating from decondensing granules (red arrow). Adapted from reference [7]. 